\title{
Design and development of installation of the electromechanical transformer from a discrete secondary part for cellulose grind process
}

\author{
Denis N. Kuimov ${ }^{1, *}$, and Maxim S. Minkin ${ }^{1}$ \\ ${ }^{1}$ Don State Technical University, Department of automation of production processes, 344000 \\ Rostov-on-Don, Russia
}

\begin{abstract}
In the present publication, problems of design of the electromechanical transformer with a discrete secondary part are considered. Design process is analysed, the key geometrical parameters of a tooth zone of the inductor are calculated. Process of an optimization of the inductor is carried out.
\end{abstract}

\section{Introduction}

Now the large volume of researches in the field of development of similar devices for an intensification of processing of liquid materials is conducted. However all these works have separate character and solve separate, private problems, without allowing designing effective installation. The majority of installations with the discrete working part moving under the influence of an external magnetic field are subject to existence of dead bands. These zones appear in the field of the working chamber where there is no heavy traffic of ferromagnetic elements.

Therefore, the purpose of the present of work is development of a technique of design of installation of the electromechanical transformer with a discrete secondary part for cellulose grind process implementation.

\section{Main part}

The design procedure of any electrical machine comes down to implementation of development stages of construction of the inductor, electromagnetic and thermal calculations. In the real work, within an objective application of a configuration of a magnet system of the device with the multiphase inductor with the concentrated coils is supposed.

The inductor is the electromechanical transformer with a discrete secondary part (ETDSP) main module. According to analogy to inductor machines, the main and major stage the choice of number of phases is $[1,2]$. The number of phases of the inductor ETDSP has significant effect on overall performance of the device. Not only power and technical

\footnotetext{
* Corresponding author: kuimov d@list.ru
} 
and economic indicators of the device, but also technological complexity of production depend on number of teeth of the inductor.

In terms of functioning of ETDSP, process of the movement of big set of ferromagnetic elements substantially is defined by number of phases of the inductor. The theoretical analysis of works, allows continuing a further research on the assumption of increase in number of phases. In case number of phases more than 3 that at any position in space of a ferromagnetic element there will be a coil or pair of coils of a magnet system at which excitation the body will start moving. At the same time elimination of so-called "dead" zones of the 1st order in the field of corners of the teeth of the inductor which are available in the previous configurations of devices as with the distributed winding, and configurations with concentrated winding and small number of poles is provided.

The physical phenomenon which is shown that on each unit ferromagnetic element, in an electromagnetic field electromagnetic attracting force which aims to move a ferromagnetic element to an active pole, that is to the area with the greatest intensity of a field works is the cornerstone of an operation principle of the offered configuration of ETDSP.

In terms of functioning of ETDSP, the nature of the movement of ferromagnetic elements significantly reacts to change of number of phases. In work [3] transition from the inductor with the distributed winding to the inductor with concentrated winding and the number of poles equal is shown we rub. The results of modelling of the device presented in work with a discrete secondary part revealed a problem of existence of a "dead" zone of the 1 st order in an intertooth zone in which more than $50 \%$ of work ferromagnetic items "stuck". Big intertooth zones and considerable differences of values of a magnetic flux density different removal from an active pole explain the negative effect. Elements get to an intertooth zone in which the value of a magnetic flux density can be 10 times less values of a magnetic flux density in the field of poles. As a result, for movement of the elements which got to an intertooth zone, insufficiently electromagnetic field of the excited pole and they stop the movement. In order that a discrete secondary part freely and rather smoothly moved along boring of the inductor, without meeting on the way of relocation of areas with considerable differences of a magnetic flux density, it is necessary to reduce the value of an intertooth zone increase in number of teeth.

Leaning on theoretical researches, it is possible to draw a conclusion that it is the most rational to build a technique of design of ETDSP on the basis of a research of process of the movement of a discrete secondary part as the most important factor characterizing overall performance of the device. In the course of the analysis of configurations of magnet systems parameters of the electromagnetic effort, operating on a ferromagnetic element on different removal from the excited pole will be considered as key indicators.

The sequence of the offered technique of design of the inductor ETDSP includes the following actions:

1) Choice of a configuration of a magnet system of the inductor;

2) Calculation and optimization of parameters of a magnet system of the device;

3) Research of the thermal mode;

4) Choice of an algorithm of inclusion of phases of the inductor.

At the first stage for receiving initial geometry of the inductor of the electromechanical transformer, predesign based on application of analytical techniques of design is carried out [4]. At this stage, the number of initial input data is minimum.

At design of the inductor, it is convenient to proceed from inside diameter. In our case the inside diameter of the inductor is set as, based on experience of developments of similar devices for the industrial enterprises, defines effective distribution of a magnetic flux density in the working chamber and optimum "live" section. 
For determination of parameters of construction of the inductor actions, in many respects similar to the maintenance of a method of calculation of construction of a magnet system of inductor machines were carried out [5].

Assessment of nature of change of magnetic field in the working chamber and its influences on overall performance of EPDVCh is carried out using a mathematical model on the basis of the multiphysical program Ansys Maxwell complex. The program complex reshayushcht problems of calculation of an electromagnetic field on the basis of use of Maxwell equations for finite regions of spaces with the corresponding boundary conditions [6]:

$$
\begin{aligned}
& \operatorname{rot} \bar{H}=\bar{J} \\
& \operatorname{div} \bar{B}=0, \\
& \bar{H}=\mu \mu_{0} \bar{H},
\end{aligned}
$$

where - a vector of magnetic field strength, - a current density vector, - relative magnetic permeability, - a magnetic constant.

Laplace-Poisson's equation of relative magnetic vector potential takes the following form [41]:

$$
\frac{1}{\mu} \nabla^{2} \bar{A}=-\mu_{0} \bar{J}
$$

In a three-dimensional coordinate system (xyz) magnetic field has three making vectors of magnetic potential, therefore, the equation (2.11) will take a form:

$$
\begin{aligned}
& \frac{1}{\mu} \nabla^{2} A_{x}=-\mu_{0} J_{x} \\
& \frac{1}{\mu} \nabla^{2} A_{y}=-\mu_{0} J_{y} \\
& \frac{1}{\mu} \nabla^{2} A_{z}=-\mu_{0} J_{z} .
\end{aligned}
$$

The solution of this system of equations in the Ansys Maxwell software package is complemented with boundary conditions. Ansys Maxwell is one of the most widespread software packages of modeling of electromagnetic fields and a research of two-dimensional and three-dimensional models, based on the finite-element method (Finite Element Method - FEM) [7].

Distinctive feature of a magnet system with concentrated winding with number of poles more than six is the possibility of implementation of movement of ferromagnetic elements on a circle along boring of the inductor without "sticking" in an intertooth zone.

The overall performance of projectible ETDSP is defined by quantity of the work ferromagnetic items involved in the general movement. At the same time the selected configuration of the inductor should provide such distribution of an electromagnetic field in the work area at which perhaps full elimination of a "dead" zone of the 1 st order. Calculation results are presented in table 1. 


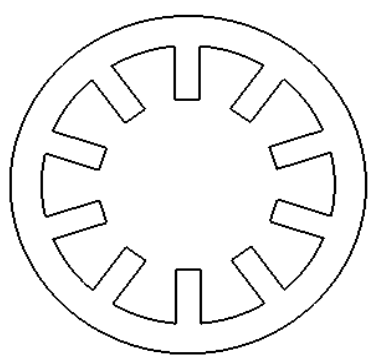

Fig. 1. Studied inductor configurations.

Table 1. Calculation results

\begin{tabular}{|l|l|}
\hline Name of parameter & Value of parameter \\
\hline Number of poles of inductor & 10 \\
\hline Number of phases & 10 \\
\hline Outside radius of the inductor, $\mathrm{mm}$ & 90 \\
\hline Inner radius of the inductor, $\mathrm{mm}$ & 40 \\
\hline Height of a tooth, $\mathrm{mm}$ & 29 \\
\hline Width of a tooth, $\mathrm{mm}$ & 12 \\
\hline
\end{tabular}

\section{Optimization of the tooth zone}

Today, there is a set of different techniques of design of a magnet system of ETDSP, however they do not use fully a possibility of algorithms of optimization. It is necessary to add that still research problems of devices were in most cases solved with a discrete secondary part experimentally, by measurement of a magnetic flux density on the volume of the working chamber. At the same time optimum design is the difficult multiple task demanding accounting of a set of criteria. For improvement of parameters of a magnet system of ETDSP it is necessary to use optimization methods.

Optimization plays a key role at design of any devices including ETDSP, and can be made by different criteria: cost value minimum; minimum of a material capacity of the device (active part); a minimum of thermal losses in a magnetic conductor and in windings; maximum of electromagnetic effort (electromagnetic moment); and many other criteria, depending on the specification. Optimization execution at the same time by several criteria is also possible. Besides, improvement of characteristics of the device can be received by carrying out optimization by different criteria with the subsequent analysis of results and the choice of the most suitable.

Construction of the inductor ETDSP at the same time should meet several requirements, many of which can be contradictory. For example, providing simpler conditions of elimination of "dead" zones demands reduction of inner radius of the inductor that in turn reduces the mass of the inductor, reduces winding volume, but at the same time leads to significant reduction of throughput of the device. Or increase in MDS of a winding for increase in the electromagnetic effort operating on a work ferromagnetic item leads to increase in power consumption and increase in volume of a winding.

Problem definition of optimization of a tooth zone of the inductor can be considered correct only in that case when criteria and parameters of optimization are specified and proved and restrictions are formulated [8].

In this case the combined approach of parameter optimization was selected $[9,10]$. The geometrical sizes of the inductor of the device act as the optimized parameters. For a solution of a problem of optimization of the inductor ETDSP first of all it is necessary to 
decide on the optimality criterions which are functions from optimization parameters. As parameters of optimization, the geometrical sizes of tooth zone ETDSP were accepted (figure 2): inductor b1 tooth crown height; inductor b2 tooth width; b3 tooth crown width.

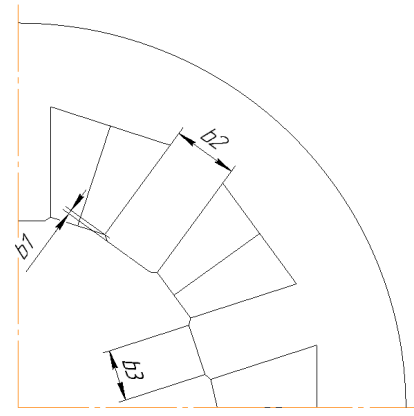

Fig. 2. Cross-section of initial geometry of a tooth zone of the inductor of the device.

For these parameters, it is necessary to write mathematical expression of target function. The efficiency of the electromechanical transformer with a discrete secondary part is estimated first of all by efficiency of the movement of ferromagnetic elements on the working chamber. Earlier it was noted that the efficiency of the movement of elements is determined by the nature of distribution of magnetic field by the working chamber, with an average a magnetic flux density on the volume of the working chamber of 0.15-0.2 $\mathrm{T}$ [9]. However often achievement of the set range of change of a magnetic flux density does not provide the optimum movement of secondary elements that is confirmed by results of scientific works [17]. Therefore as key parameter the average electromagnetic effort influencing from the created electromagnetic field unit ferromagnetic work item on all volume of the working chamber is selected. A condition of restriction of admissible heating of steel of the inductor and according to a condition of restriction of a magnetic flux density in a magnetic conductor will be additional parameters of optimization. Thus, most good results by optimization of a tooth zone of the inductor of the electromechanical transformer with a discrete secondary part can be achieved, only using multicomponent target function of the following look:

$$
\mathrm{F}_{\mathrm{c}}=\mathrm{k}_{1}\left(\frac{\mathrm{F}_{\mathrm{mc}}-\mathrm{F}_{\mathrm{mt}}}{\mathrm{F}_{\mathrm{mc}}}\right)+\mathrm{k}_{2}\left(\frac{\mathrm{B}_{\mathrm{c}}-\mathrm{B}_{\mathrm{t}}}{\mathrm{B}_{\mathrm{c}}}\right)+\mathrm{k}_{3}\left(\frac{\mathrm{t}_{\mathrm{c}}-\mathrm{t}}{\mathrm{t}_{\mathrm{c}}}\right)+\mathrm{k}_{4}\left(\frac{\mathrm{B}_{\mathrm{tc}}-\mathrm{B}_{\mathrm{tT}}}{\mathrm{B}_{\mathrm{tc}}}\right) \rightarrow \mathrm{min},
$$

where, k1, k2, k3, k4 - weighting coefficients for each criterion of optimization; Fmc target mean value of the electromagnetic effort operating on unit ferromagnetic element from an electromagnetic field, N; Fmt - the current mean value of the electromagnetic effort operating on unit ferromagnetic element from an electromagnetic field, $\mathrm{N}$; $\mathrm{Bc}-$ the target relation of values of a magnetic flux density on the volume of the working chamber, $\mathrm{T}$; $\mathrm{Bt}$ - the current relation of values of a magnetic flux density on the volume of the working chamber, $\mathrm{T}$; $\mathrm{tc}-$ target value of temperature of steel of a magnetic conductor, ${ }^{0} \mathrm{C} ; \mathrm{t}$ - the current value of temperature of a magnetic conductor, ${ }^{0} \mathrm{C}$; Btc - target value of a magnetic flux density in steel of a magnetic conductor, $\mathrm{T}$; BtT - the current value of a magnetic flux density in steel of a magnetic conductor, $\mathrm{T}$.

Values of weighting coefficients are selected depending on degree of the importance of a separate component of criterion of optimization. The sum of weighting coefficients always should equal 1 . 
Except a task of target function, it is necessary to select the method allowing to pick up the optimum geometrical sizes of a tooth zone of the inductor of the electromechanical transformer for rather small period. Analysing scientific works on problems of optimization [43-46] and also experience of design and development of inductors of electromechanical transformers, among a large number of algorithms of optimization, good results at a solution of a goal were received using a stochastic algorithm Monte Carlo (a method of random numbers).

The essence of the Monte-Carlo method consists in search of a required extremum of target function by the accidental choice of the geometrical parameters defining a magnet system of the inductor of the electromechanical transformer. In practice this method means carrying out $\mathrm{N}$-go of number of tests as a result of which receive $\mathrm{N}$ possible values of required function. On the basis of it the conclusion about a high error of the selected method which value is inversely proportional to the number of tests [43] is drawn. From this it follows that the high number of tests will allow to achieve the high accuracy of calculations. At an initial stage the choice of accidental parameters was made with uniform distribution in the field of permissible values, and then were specified with normal distribution of a random variable. Best values of height and width of a tooth and also tooth crown width were selected. The vector of optimization of $X=(b 1 \ldots b 1 n, b 2 \ldots b 2 n, b 3 \ldots$ b3n, I) where b1, b2, b3, I - the tooth height, tooth width, tooth crown width, value of a phase current was as a result created.

Earlier it was shown that as parameters of optimization four ETDSP parameters are used. For execution of the procedure of optimization it is necessary to define limits of change of each parameter. Range of change of parameters in the course of optimization is in the following limits:

1) From 0 to $10 \mathrm{~mm}$ - tooth crown width range;

2) From 8 to $15 \mathrm{~mm}$ - tooth width range;

3) From 0 to $4 \mathrm{~mm}$ - tooth crown height range;

4) From 10 to 30 And - phase current range.

For determination of numerical value of target function it is necessary to carry out electromagnetic calculation. On the basis of the carried-out analysis of implementers of a mathematical model of the main software packages allowing to carry out calculation of electromagnetic devices (MatLab, Ansys, ELCUT, FEMM) as coding environment for execution of computing experiments with the electromechanical transformer with a discrete secondary part also used the Ansys software package.

Upon transition directly to a solution of an optimization task it is necessary to set starting values of geometrical parameters of a tooth zone of the inductor which need to be changed. At this stage the initial geometrical sizes of a tooth zone on the basis of the carried-out earlier analytical calculation were selected:

1) Width of a tooth is $12 \mathrm{~mm}$;

2) Height of a crown of a tooth of $12 \mathrm{~mm}$;

3) Width of a crown of a tooth of $1 \mathrm{~mm}$.

Diameter of the working chamber is basic parameter and is selected on the basis of experience of application of ETDSP in processing of liquid environments and cannot change in the course of optimization, entering restrictions for changes of parameters of a tooth zone.

Height of a tooth of the inductor is connected with diameter of the working chamber and inside diameter of the inductor. Therefore, tooth height value also remains invariable in the course of the procedure of optimization of a tooth zone. The transverse section of the inductor and working chamber defining outline borders of estimated area is given in figure 3.

At creation of a design model, the following assumptions were accepted: 
1) The arrangement of ferromagnetic elements is chaotic and chaotic, and fixed for all experiments;

2) Phases are independent from each other in the magnetic relation;

3) Active and inductive resistance of each phase are equal to active and inductive resistance of other phases;

4) Current density in the section of a winding is distributed evenly.

In ETDSP the electromagnetic field exists in the following set of environments:

1) Ferromagnetic core of the inductor (brand 2214 steel);

2) The processed liquid environment - oil, being low-magnetic material, within calculations it was replaced with air;

3) The areas occupied with conductors with current;

4) Ferromagnetic elements.

\section{Analysis of physical processes}

The research of samples after cavitational processing revealed good fibrillation of a surface of fibers. Their ends were subject to the greatest processing. Authors [11] specified lack of deep destructions of fibers, increase in flexibility and plasticity that is important for receiving high physicomechanical rates.

According to a number of researchers [12], the destroying impact of cavitational processing is a consequence of blows of high-speed micro streams and impulses of pressure of the bubbles arising at short circuit. Repeated impact of shock waves on material leads to emergence in it fatigue stresses, and then and deformations. In view of the fact that the range of micro streams is small, they render effective effort to fiber only at a collapse of bubbles in close proximity to a surface.

The main operating part of devices of this type are the kavitator of different configurations set permanently in a flow of suspension or fixed to the rotating shaft in the form of plates of a trapezium form, turned big the basis towards rotation. For gain of effect counterkavitator can be installed in the camera.

Power expenses of this way of processing of fiber still considerable. Despite good strength characteristics of castings from weight not only coniferous, but also deciduous breeds of wood, it was not widely used in the industry.

For reduction in cost of receiving nanocellulose process of impact of hydrodynamic cavitation in the electromechanical transformer with a discrete secondary part is offered. The main operating part of devices of this type is the big set of the small ferromagnetic elements representing the working secondary discrete part moving under the influence of an outside electromagnetic field.

Power expenses of this way very insignificant and caused only by power costs of movement of elements on the set trajectory depending on an algorithm of switching of phases of the inductor.

\section{Conclusion}

The presented work is devoted to questions of modelling, a research and optimum design of the electromechanical transformer with a discrete secondary part intended for cellulose suspension grind process implementation. Practical value consists in development of a technique of design of installations on the basis of a research of influence of an electromagnetic field on ferromagnetic elements. The presented technique in the future will simplify development process of new installations for satisfaction of needs of vendors.

The reported study was funded by RFBR according to the research project № 18-29-18064. 


\section{References}

1. V.A. Smiths, Electricity, 10, 24 (2007)

2. A.B. Krasovsky, Vestnik MSTU of N.E. Bauman. Mechanical engineering series, 4 (2005)

3. E.A. Klimov, V.V. Kolosov, V.E. Saprykin, News of higher educational institutions. Electromecanics, Novocherkassk, 1, 67 (2012)

4. V.N. Galushko T.V. Alferova, S.I. Bakhur, A.A. Alferov, Messenger of the Gomel state technical university of P.O. Sukhy, 3, 45 (2014)

5. V.G. Fisenko, A.N. Popov, Design of valve inductor engines Methodical grant at the rate "Special Electrical Machines" (Publishing house MAI, Moscow, 2005)

6. E.G. Andreyeva, A.A. Tatevosyan, I.A. Syomina, Omsk scientific bulletin, 2(120), 231 (2013)

7. A.S. Martyanov, N.I. Neustroyev, International scientific log alternative power engineering and ecology, 19(159), 47 (2014)

8. A.V. Shevkunova, New science: Problems and perspectives, 3-2, 248 (2016)

9. A.V. Kashuba, Works of the Rostov state transport university, 3, 62 (2016)

10. N.N. Klochkova, A.V. Obukhova, Bulletin of the Samara state technical university. Series: Technical science, 2, 161 (2010)

11. Yu.D. Alashkevich, V.A. Boyarchenko, V.G. Vasyutin, N.V. Ovchinnikov, Modern machines and devices of chemical productions: Proc. the third All-Union. scientific conf. Part VI, Tashkent, 59 (1983)

12. A.E. Geguzin, Drop (Science, Moscow, 1977)

13. G.K. Ivanitsky, Collection of scientific articles Modern science, 2011, 2(7), 52 (2011) 